\title{
In Vivo Assesment of Anti-trypanasomal Effect of Rosemary (Rosmarinus officinalis) In Rabbits
}

\author{
${ }^{1 *}$ Fathy Mohamed Ali Awad, ${ }^{2}$ Mohamed Abdelrahman Mohamed, ${ }^{2}$ Abdinasir \\ Yusuf Osman, ${ }^{3}$ Zainal Abidin Abu-Hassan, and ${ }^{1}$ Nazlina Ibrahim \\ ${ }^{I}$ School of Biosciences and Biotechnology, Universiti Kebangsaan Malaysia, 43600 UKM Bangi, Selangor, \\ Malaysia. \\ ${ }^{2}$ Faculty of Veterinary Medicine, Universiti Putra Malaysia, 43400 UPM Serdang, Selangor, Malaysia \\ ${ }^{3}$ Faculty of Medicine, Universiti Teknologi MARA UiTM, Shah Alam Selangor, Malaysia.
}

\begin{abstract}
Today, tropical diseases pose significant risk to animal and human populations worldwide. Most of which were classified as neglected tropical diseases, having an enormous impact on socio-economic development and quality of life at all levels particularly in developing countries. At present, a lot of research is committed to sleeping sickness, not only because they are major killing diseases but also because disease control becomes more difficult due to a number of factors that limit the utility of current drugs in resource-poor settings, such as high cost, poor compliance, drug resistance, low efficacy and poor safety. Hence, the search for new and preferably cheap drugs needs to be continued.

Natural products are still major potential sources of innovative therapeutic agents for various conditions, including infectious diseases as they represent an unmet source of chemical diversity. This study was conducted in order to determine the anti-trypanosomal properties of crude chloroform extract (CCE) of Rosmarinus officinalis. A total of thirty rabbits $(n=30)$ of about $5-6$ months old, weighing $1.5-2.5 \mathrm{~kg}$ were enrolled and divided into six main groups of five animals each. The course of Trypanosoma evansi infection in rabbits was followed for 48 days post-infection. Animals were closely observed for clinical examination. Blood samples were taken to measure haematological and biochemical parameters. Our results revealed abnormalities in all investigated parameters in infected group. In contrast, animals treated with CCE of Rosmarinus officinalis showed normalization in their haematological and biochemical values thereby confirming their anti-parasitic properties.
\end{abstract}

Keywords: chloroform extracts of Rosmarinus officinalis, Trypanosoma evansi, Parasitaemia levels, haematological and biochemical values, rabbits.

\section{Introduction}

Trypanosoma evansi is a protozoa that causes Trypanasomiasis in animals and humans and it belongs to theTrypanosomatidae family. Trypanosoma evansi is the most commonly distributed of the pathogenic African animal trypanosomes, affecting domestic livestock and wildlife in Asia, Africa and Latin America (Luckins, et al., 2004).

Trypanosomiasis is a disease of great economic importance in livestock in the world because it affects important working animals such as: Buffalo, cattle, camels, horses and other animals, including wildlife, can also be infected (OIE, 2008).

Despite the scientific evidence published in the database, yet, $T$. evansi has not been included in the animal health conditions for international trade in many countries across the globe (Gutierrez et al., 2000). Strategic controlling has mostly focused on the use of chemotherapy options as anti-trypanosomal medicines. However, extensive resistance of Trypanosoma spp to these drugs has developed. As an alternative, research is being conducted to identify plant products with anti-trypanosomal properties.

Recently, the use of medicinal plants and herbs has increased throughout the world for the maintenance and improvement of health and for the treatment of various human conditions and diseases.

Rosmarinus officinalis, popularly known as rosemary is a woody, perennial herb with fragrant, evergreen, needle-like leaves and white, pink, purple, or blue flowers, native to the Mediterranean region. It belongs to the family Lamiaceae, genus Rosmarinus which includes many other herbs.

It is used as carminative, rubifacient, stimulant and as flavouring agent for liniments, hair lotions, inhaler,soaps and cosmetics (Kokate et al. 2010). Rosemary leaves have many traditional uses based on their antibacterialand spasmolytic actions. Used orally for the treatment of dyspeptic complaints [British Herbal...1996), and in external applications for supportive management of rheumatic complaints and circulatory disorders[Blumenthal 1998].Medicinally, Rosmarinus officinalis has antibacterial, anti-mutagenic properties, chemopreventive agent (Oluwatuyi, et al., 2004). And antioxidant activity (Peng, et al., 2005). It is used as 
fresh, dried or as the essential oil (bauret al., 1990)It was reported that the plant has antiplasmodial activity to protect malaria.

\section{Plant Material and the Extract Preparation}

\section{Materials and Methods}

The aerial parts of Rosmarinus officinalis herbs were collected during the flowering and vegetative phase of the plant at different localities characterized by diverse geographic and climate conditions in Libya.The plants were cut at ground level and those portions from above ground were air dried for 2 weeks in a laboratory of the Botany Department, Omar Al Mukhtar University, Al Beida, Libya. The chopped, air dried plant material was stored in refrigerator, and awaited for extraction analysis. Subsequently, the crude chloroform extract was prepared at the Faculty of Science and Technology, Universiti Kebangsaan Malaysia (UKM), Bangi. Dried plants of R.officinalis grounded into powder. By using a Soxhlet apparatus with $95 \%$ chloroform at a temperature of $50^{\circ} \mathrm{C}$ for $12 \mathrm{~h}$, five hundred gram powdered plant material was extracted. The residue was removed by filtration. The extract was concentrated in a rotary evaporator under reduced pressure at temperature of $40-50{ }^{\circ} \mathrm{C}$ and then lyophilized to obtain a powdered extract and stored at $4{ }^{\circ} \mathrm{C}$ until used (Eneet al., 2009).

\section{Animals}

The study was approved by the Ethics committee of UKM (UKMAEC) for the Care and Use of Animals. Experiments utilized 5 - 6 months old, New Zealand clinically healthy rabbits. All rabbits $(n=30)$ were screened for the presence of haemo-protozoan parasites using wet mount and leishman stained blood films after arrival and were all confirmed negative. The animals were maintained in a fly proof isolation unit at the experimental animal house, Faculty of Biosciences and Biotechnology, Universiti Kebangsaan Malaysia (UKM) inside large appropriate metal cages (1 per cage). Rabbits were provided ad libitum access to water and a stock chow pellets throughout the experimental period.

\section{Stock of Parasites and Inoculum preparation}

The Trypanosoma evansi isolate used for the experiment was taken from the Parasitology Laboratory, Faculty of Veterinary, Universiti Putra Malaysia (UPM). The parasite was originally isolated from a naturally infected deer from Perak state, Malaysia in 2007 (Adrian et al., 2010). The isolate is propagated by subpassaging in mice. An inoculum of $5 \times 10^{5}$ parasites in $1 \mathrm{ml}$ was then prepared by diluting the pooled mice blood with Alsever's solution.

\section{Experimental procedure}

Rabbits were divided into 6 major groups of 5 rabbits in each group at the start of the experiment (Fig. 1). Animals of Group 1 were pre-treated for two days intraperitoneally with $20 \mathrm{mg} \mathrm{kg}^{-1}$ of crude chloroform extract (CCE) of R.officinalis before inoculation with T. evansi and thereafter treated for 6 days after infection. Rabbits of Group 2 were treated intraperitoneally with $20 \mathrm{mg} \mathrm{kg}^{-1}$ of CCE and concurrently infected with $T$. evansi. Rabbits of Group 3 were treated 6 days with $20 \mathrm{mg} \mathrm{kg}^{-1}$ of CCE for 6 days after establishment of parasitemia. Animals in Group 4 positive control were treated once with $3.5 \mathrm{mg} \mathrm{kg}$-1 of Berenil® (Diminazeneaceturate) after the establishment of parasitemia. Rabbits in Group 5 were only infected once intraperitoneally with $1 \mathrm{ml}$ of $5 \times 10^{5}$ parasites. Animals of Group 6 served as reference group and were injected intraperitoneally with $1 \mathrm{ml}$ of sterile Alsever's solution. The detailed information of the experimental design was summarized in Fig. 1.

\section{Estimation of parasitemia}

All the Rabbits were sampled at two days interval during 48 days post infection (pi), about $2 \mathrm{ml}$ of peripheral blood were collected from the rabbit's marginal ear vein after shaving the area. Parasitaemia count was estimated by Micro- Haematocrit Centrifugation Technique (MHCT) (Woo, 1970). About $75 \mu 1$ of fresh blood were taken with a heparinized capillary and centrifuged for $5 \mathrm{~min}$ at $12,000 \mathrm{~g}$. Using a light microscope (100 or $400 \mathrm{X}$ magnification) capillary tubes were examined for detection and counting trypanosomes when the numbers were few around the buffy coat plasma interphase area (Woo, 1970). High parasitemia enumeration was undertaken using a Neubauer hemocytometer.

\section{Collection of blood samples}

Blood samples for haematological and biochemical analyses were collected by marginal ear venous puncture once every two weeks until 48 days post-infection. Blood samples for hematology were collected into tubes containing anticoagulant, ethylene diaminetetriacetic acid (EDTA). Blood samples for biochemical analyses were collected into tubes containing no ethylene diaminetetriacetic acid as anticoagulant. 


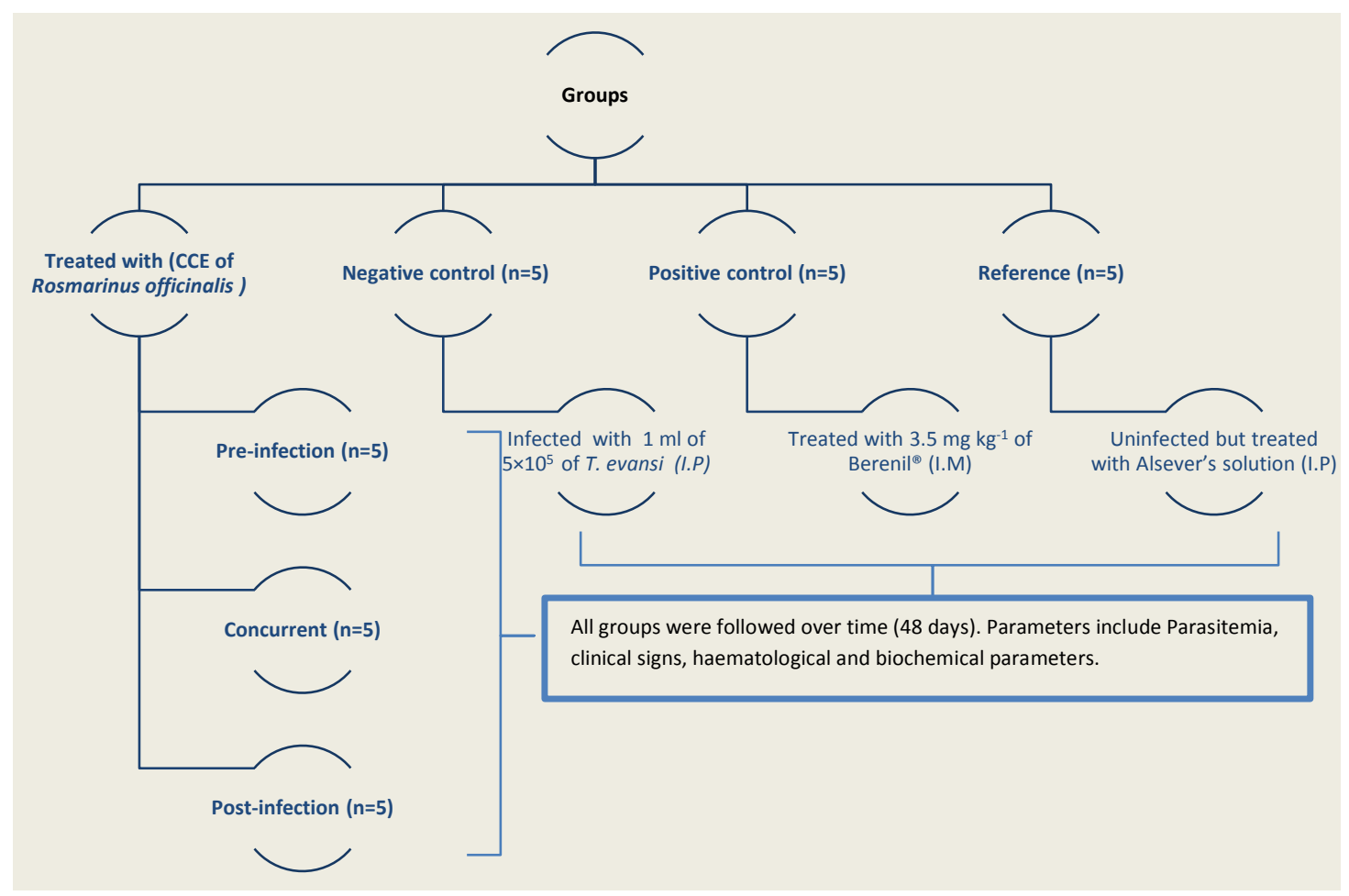

Fig. 1 Diagrammatic Representation of Experimental design

\section{Clinical examination}

At the start of the study until the end point of the experiment, the clinical observations presented by the diseased and non-diseased groups were regularly documented. Because the scoring systems require capture of signs and symptoms since the beginning of the experiment, the information collected from the 6 groups was based on the individual presentation of the clinical signs. In summary, the clinical signs of 6 groups were scored in scale of 0-3 based on the presence of following parameters: loss of condition, oedema of the face, ocular discharges, encrustation of the lips and mortality rate. The score 0 represented no abnormality of clinical signs observed, 1 for mild (30\% abnormality), 2 for moderate (60\% abnormality), 3 for severe (more than $60 \%$ abnormality).

\section{Estimation of haematological examination}

The haematological parameters that were examined include Packed cell volume (PCV), haemoglobin concentration $(\mathrm{Hb} \%)$, mean corpuscular volume $(\mathrm{MCV})$ and mean corpuscular haemoglobin concentration (MCHC) and differential leucocytic count. They were measured in heparinized blood according to Jain (2000). (Jain, N.C., 2000. Schalm's Veterinary Hematology, 4th ed. LEA and Fibiger, Philadelphia, USA.)

\section{Estimation of biochemical assays}

By using automated chemistry analyzer (HITACHI 902 Automatic Analyzer®, Japan), changes of alkaline phosphatase (AP), alanine amino transferase (ALT), aspartate amino transferase (AST), and plasma glucose activities were measured.

\section{Statistical methods}

Evaluation of the obtained results were performed by using The statistical package Version JMP 9, SAS, where the Values of Analysis of Variance (ANOVA) were used to detect the significant changes among the experimentally infected Rabbits.

\section{Parasitological examination}

\section{Results}

Daily examination of the blood of the five inoculated rabbits (1-5)by BCT showed that T. evansi started to appear on the 10 days dpi. The patent period was 5 days in all the inoculated rabbits. At 14 days pos-infection (DPI), maximum parasitaemia was observed with all 5 animals in each group being positive for T. evansi. After 
14 days post-infection, periods of low parasitaemia were intermixed with aparasitaemia periods in rabbits of experimental groups 1-4(Fig. 2). The reference group showed no $T$. evansi during the experimental period. During the first two weeks dpi, the infection in rabbits was slightly pathogenic in treated groups. These animals showed mild symptoms appearing from day 1- 9 dpi. In contrast, animals in group 5 (negative control), parasitaemia was recorded from day 6 post-infection where animals remained with the high peaks of parasitaemia between day 10-45 post-infection showing severe symptoms in the investigated parameters.

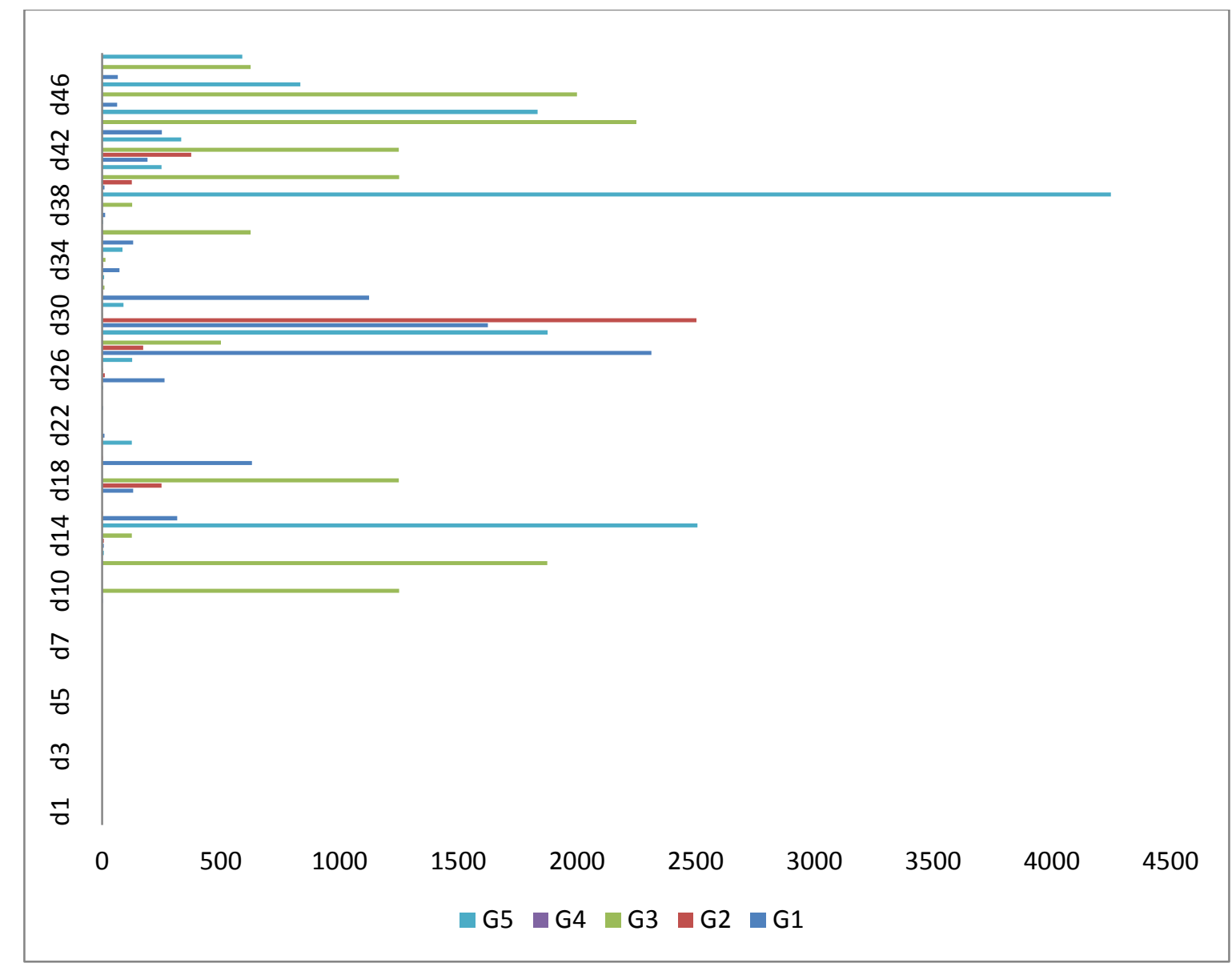

Fig.2 Parasitaemia of T. evansi from groups (1-5) involved in the study

\section{Clinical Observation}

The results obtained from the clinical observation were outlined in Tables 1-4. Animals served as negative control were severely affected after experimental infection with $T$. evansi. In these animals, the clinical signs due to the infection of $T$. evansi appeared on day 3 post-infection where most of the animals exhibited typical clinical signs that included oedema in the face, ocular discharges, poor condition and encrustation of the lips. The mean ranks of these tested parameters were significantly higher than all treated groups and control group (Table 1-4). In contrast, these clinical signs were prevented by intraperitoneal administration of R.officinalis in groups 1-3 throughout the experimental period. Similarly, animals treated with the standard drug (Berenil $\left.{ }^{\circledR}\right)$ showed no clinical signs for the whole duration of the experimental period. No significant difference was observed between chloroform extracts treated and Berenil ${ }^{\circledR}$ treated groups. 
Table 1: Mean rank of poor condition between reference group, treated groups with CCE of Rosmarinusofficinalisand negative group

\begin{tabular}{lllllll}
\hline \multirow{2}{*}{ DPI } & \multicolumn{5}{c}{ Parameters } \\
\cline { 2 - 6 } & Reference & Pre-infection & Concurrent & Post-infection & Positive control & Negative Control \\
\hline & & & & & \\
$\mathbf{2}$ & $0.0^{\mathrm{a}, \mathrm{x}}$ & $1.5^{\mathrm{b}, \mathrm{x}}$ & $1.5^{\mathrm{b}, \mathrm{x}}$ & $1.5^{\mathrm{b}, \mathrm{x}}$ & $1.5^{\mathrm{b}, \mathrm{x}}$ & $3.0^{\mathrm{c}, \mathrm{z}}$ \\
$\mathbf{1 0}$ & $0.0^{\mathrm{a}, \mathrm{x}}$ & $1.0^{\mathrm{a}, \mathrm{y}}$ & $1.5^{\mathrm{b}, \mathrm{x}}$ & $1.0^{\mathrm{a}, \mathrm{y}}$ & $1.0^{\mathrm{a}, \mathrm{y}}$ & $2.5^{\mathrm{c}, \mathrm{z}}$ \\
$\mathbf{2 0}$ & $0.0^{\mathrm{a}, \mathrm{x}}$ & $1.5^{\mathrm{b}, \mathrm{x}}$ & $1.5^{\mathrm{b}, \mathrm{x}}$ & $1.0^{\mathrm{a}, \mathrm{y}}$ & $1.0^{\mathrm{a}, \mathrm{y}}$ & $2.0^{\mathrm{b}, \mathrm{y}}$ \\
$\mathbf{3 0}$ & $0.0^{\mathrm{a}, \mathrm{x}}$ & $1.5^{\mathrm{b}, \mathrm{x}}$ & $1.5^{\mathrm{b}, \mathrm{x}}$ & $1.0^{\mathrm{b}, \mathrm{y}}$ & $1.5^{\mathrm{b}, \mathrm{x}}$ & $3.0^{\mathrm{c}, \mathrm{z}}$ \\
$\mathbf{4 0}$ & $0.0^{\mathrm{a}, \mathrm{x}}$ & $1.0^{\mathrm{b}, \mathrm{y}}$ & $1.0^{\mathrm{b}, \mathrm{y}}$ & $1.0^{\mathrm{b}, \mathrm{y}}$ & $1.0^{\mathrm{b}, \mathrm{y}}$ & $1.5^{\mathrm{b}, \mathrm{y}}$ \\
\hline
\end{tabular}

*DPI: Days post-infection

${ }^{a-c}$ Means with different superscripts within row differed significantly $(p<0.05)$ due to treatment effect.

${ }^{\mathrm{x}-\mathrm{z}}$ Means with different superscripts within column differed significantly $(\mathrm{p}<0.05)$ due to time effect.

Table 2: Mean rank of oedema between reference group, treated groups with CCE of Rosmarinus officinalis and negative group

\begin{tabular}{llllccc}
\hline \multirow{2}{*}{ "DPI } & \multicolumn{5}{c}{ Parameters } \\
\cline { 2 - 7 } & $\begin{array}{l}\text { Referen } \\
\text { ce }\end{array}$ & Pre-infection & Concurrent & Post-infection & Positive control & Negative Control \\
\hline & & & & & $1.0^{\mathrm{a}, \mathrm{x}}$ & $2.5^{\mathrm{c}, \mathrm{z}}$ \\
$\mathbf{2}$ & $0.0^{\mathrm{a}, \mathrm{x}}$ & $1.5^{\mathrm{b}, \mathrm{y}}$ & $1.5^{\mathrm{b}, \mathrm{x}}$ & $1.0^{\mathrm{a}, \mathrm{x}}$ & $1.5^{\mathrm{b}, \mathrm{y}}$ & $2.0^{\mathrm{b}, \mathrm{y}}$ \\
$\mathbf{1 0}$ & $0.0^{\mathrm{a}, \mathrm{x}}$ & $1.0^{\mathrm{a}, \mathrm{x}}$ & $1.0^{\mathrm{a}, \mathrm{x}}$ & $1.5^{\mathrm{b}, \mathrm{y}}$ & 1.0 & $2.0^{\mathrm{b}, \mathrm{y}}$ \\
$\mathbf{2 0}$ & $0.0^{\mathrm{a}, \mathrm{x}}$ & $1.0^{\mathrm{a}, \mathrm{x}}$ & $1.0^{\mathrm{a}, \mathrm{x}}$ & 1.0 & $1.5^{\mathrm{b}, \mathrm{y}}$ & $3.0^{\mathrm{c}, \mathrm{z}}$ \\
$\mathbf{3 0}$ & $0.0^{\mathrm{a}, \mathrm{x}}$ & $1.0^{\mathrm{a}, \mathrm{x}}$ & $1.0^{\mathrm{a}, \mathrm{x}}$ & $0.0^{\mathrm{a}, \mathrm{x}}$ & $1.0^{\mathrm{a}, \mathrm{x}}$ & $1.5^{\mathrm{b}, \mathrm{y}}$ \\
\hline $\mathbf{4 0}$ & $0.0^{\mathrm{a}, \mathrm{x}}$ & $1.0^{\mathrm{a}, \mathrm{x}}$ & $1.0^{\mathrm{a}, \mathrm{x}}$ & $1.0^{\mathrm{a}, \mathrm{x}}$ & & \\
\hline
\end{tabular}

"DPI: Days post-infection

${ }^{\text {a-c }}$ Means with different superscripts within row differed significantly $(\mathrm{p}<0.05)$ due to treatment effect.

${ }^{\mathrm{x}-\mathrm{z}}$ Means with different superscripts within column differed significantly $(\mathrm{p}<0.05)$ due to time effect.

Table 3: Mean rank of encrustation between reference group, treated groups with CCE of Rosmarinus officinalis and negative group

\begin{tabular}{lllcccc}
\hline \multirow{2}{*}{$*^{*}$ DPI } & \multicolumn{5}{c}{ Parameters } \\
\cline { 2 - 7 } & Reference & Pre-infection & Concurrent & Post-infection & Positive control & Negative Control \\
\hline $\mathbf{2}$ & $0.5^{\mathrm{a}, \mathrm{x}}$ & $1.5^{\mathrm{b}, \mathrm{y}}$ & $1.0^{\mathrm{a}, \mathrm{x}}$ & $1.0^{\mathrm{a}, \mathrm{x}}$ & $1.0^{\mathrm{a}, \mathrm{x}}$ & $2.5^{\mathrm{c}, \mathrm{z}}$ \\
$\mathbf{1 0}$ & $0.5^{\mathrm{a}, \mathrm{x}}$ & $1.0^{\mathrm{a}, \mathrm{x}}$ & 1.5 & $1.0^{\mathrm{a}, \mathrm{x}}$ & $1.0^{\mathrm{a}, \mathrm{x}}$ & $2.5^{\mathrm{c}, \mathrm{z}}$ \\
$\mathbf{2 0}$ & $0.0^{\mathrm{a}, \mathrm{x}}$ & $1.0^{\mathrm{a}, \mathrm{x}}$ & $1.0^{\mathrm{a}, \mathrm{x}}$ & $1.5^{\mathrm{b}, \mathrm{y}}$ & $1.5^{\mathrm{b}, \mathrm{y}}$ & $2.0^{\mathrm{b}, \mathrm{y}}$ \\
$\mathbf{3 0}$ & $0.0^{\mathrm{a}, \mathrm{x}}$ & $1.0^{\mathrm{a}, \mathrm{x}}$ & $1.0^{\mathrm{a}, \mathrm{x}}$ & $1.0^{\mathrm{a}, \mathrm{x}}$ & $1.0^{\mathrm{a}, \mathrm{x}}$ & $2.5^{\mathrm{b}, \mathrm{y}}$ \\
$\mathbf{4 0}$ & $0.0^{\mathrm{a}, \mathrm{x}}$ & $1.0^{\mathrm{a}, \mathrm{x}}$ & $1.0^{\mathrm{a}, \mathrm{x}}$ & $1.0^{\mathrm{a}, \mathrm{x}}$ & $1.0^{\mathrm{a}, \mathrm{x}}$ & $1.5^{\mathrm{b}, \mathrm{y}}$ \\
\hline
\end{tabular}

"DPI: Days post-infection

${ }^{\mathrm{a}-\mathrm{c}}$ Means with different superscripts within row differed significantly $(\mathrm{p}<0.05)$ due to treatment effect.

${ }^{\mathrm{x}-\mathrm{z}}$ Means with different superscripts within column differed significantly $(\mathrm{p}<0.05)$ due to time effect.

Table 4: Mean rank of ocular discharges between reference group, treated groups with CCE of Rosmarinus officinalis and negative group

\begin{tabular}{lllrrrr}
\hline \multirow{2}{*}{ DPI } & \multicolumn{5}{c}{ Parameters } \\
& \multicolumn{5}{c}{} & \\
& Reference & Pre-infection & Concurrent & Post-infection & Positive control & Negative Control \\
\hline $\mathbf{2}$ & $0.5^{\mathrm{a}, \mathrm{x}}$ & $1.0^{\mathrm{a}, \mathrm{x}}$ & $1.5^{\mathrm{b}, \mathrm{y}}$ & $1.5^{\mathrm{b}, \mathrm{y}}$ & $1.0^{\mathrm{a}, \mathrm{x}}$ & $2.5^{\mathrm{c}, \mathrm{z}}$ \\
$\mathbf{1 0}$ & $0.5^{\mathrm{a}, \mathrm{x}}$ & $1.0^{\mathrm{a}, \mathrm{x}}$ & $1.0^{\mathrm{a}, \mathrm{x}}$ & $1.0^{\mathrm{a}, \mathrm{x}}$ & $1.0^{\mathrm{a}, \mathrm{x}}$ & $2.5^{\mathrm{c}, \mathrm{z}}$ \\
$\mathbf{2 0}$ & $0.0^{\mathrm{a}, \mathrm{x}}$ & $1.0^{\mathrm{a}, \mathrm{x}}$ & $1.0^{\mathrm{a}, \mathrm{x}}$ & $1.0^{\mathrm{a}, \mathrm{x}}$ & $1.0^{\mathrm{a}, \mathrm{x}}$ & $2.0^{\mathrm{b}, \mathrm{y}}$ \\
$\mathbf{3 0}$ & $0.0^{\mathrm{a}, \mathrm{x}}$ & $1.0^{\mathrm{a}, \mathrm{x}}$ & $1.0^{\mathrm{a}, \mathrm{x}}$ & $1.0^{\mathrm{a}, \mathrm{x}}$ & $1.0^{\mathrm{a}, \mathrm{x}}$ & $2.5^{\mathrm{c}, \mathrm{z}}$ \\
$\mathbf{4 0}$ & $0.0^{\mathrm{a}, \mathrm{x}}$ & $1.0^{\mathrm{a}, \mathrm{x}}$ & $1.0^{\mathrm{a}, \mathrm{x}}$ & $1.0^{\mathrm{a}, \mathrm{x}}$ & $1.0^{\mathrm{a}, \mathrm{x}}$ & $1.5^{\mathrm{b}, \mathrm{y}}$ \\
\hline
\end{tabular}

*DPI: Days post-infection

${ }^{a-c}$ Means with different superscripts within row differed significantly $(p<0.05)$ due to treatment effect.

${ }^{\mathrm{x}-\mathrm{z}}$ Means with different superscripts within column differed significantly $(\mathrm{p}<0.05)$ due to time effect. 


\section{Mortality rate}

During the experimental period, no death was recorded in all treated groups (1-4) that either treated with CCE of Rosmarinus officinalis or with Berenil ${ }^{\circledR}$ (Diminazene aceturate). Similarly, animals served as reference group did not show any mortality rate in the whole duration of the experimental period. In contrast, animals within negative control (infected but not treated) started to die frequently where death of first animal (20\%) observed on day $17^{\text {th }}$. Thereafter, another 2 animals (40\%) died on day 34. The remaining 2 animals of the negative group survived throughout the experimental period making the total lethality (60\%) (Fig. 3). Mortality rates were associated with severe parasitaemia caused by the challenged strain (T.evansi).

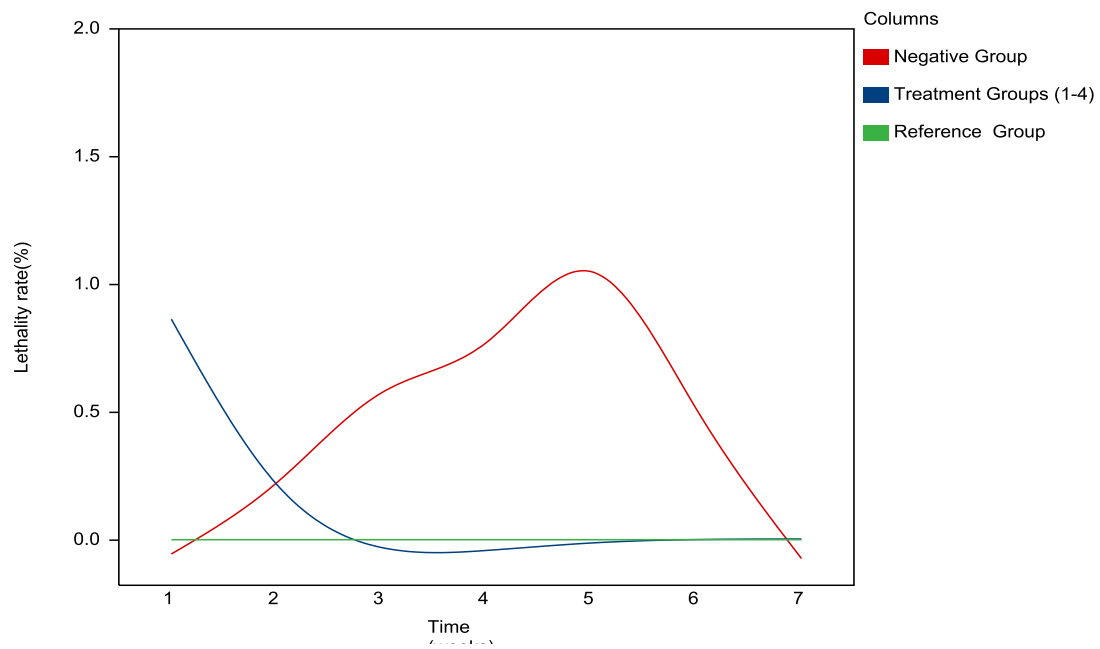

Fig. 3 Comparison of lethality rate between reference group, Treated groups with CCE of Rosmarinus officinalis and Negative group.

\section{Changes in the haematological values}

The haematological characteristics of tested parameters in the different groups involved in the study are summarized in Tables 5-12. Of these groups, animals in group 5 (negative control) showed statistically a significant decrease in the tested values of PCV, $\mathrm{Hb}$ and RBC, with maximum mean values of $(10.60 \pm 2.30)$, (74.94 \pm 7.07$),(2.46 \pm .33)$ respectively (Tables7-9). From the first week of the study, the values of these parameters started to drop and remained lower than the values of control and treated animals until the end of the experimental period. On the other hand, the values PCV, Hb and RBC of treated groups (1-4) showed slight decrease in serum levels between day $3-5$ post-infection. Thereafter, normalization of these values (PCV, RBC $\& \mathrm{Hb}$ ) was observed and remained unchanged throughout the experimental period (Table IV). There were no significant differences observed in all tested parameters in animals served as reference group (Tables 5-12).

Table 5: The effect of Crude Chloroform extract of Rosmarinus officinalis on PCV values of Rabbits infected with T. evansi

\begin{tabular}{|c|c|c|c|c|c|c|}
\hline \multirow{2}{*}{$\begin{array}{l}\text { Time } \\
\text { (Weeks) }\end{array}$} & & \multicolumn{5}{|c|}{ Groups } \\
\hline & Reference & Pre-infection & Concurrent & Post-infection & Positive control & Negative Control \\
\hline 1 & $36.00 \pm 1.73^{\mathrm{a}, \mathrm{x}}$ & $23.50 \pm 5.17^{\mathrm{b}, \mathrm{y}}$ & $25.10 \pm 3.71^{b, y}$ & $24.57 \pm 4.56^{\mathrm{b}, \mathrm{y}}$ & $28.33 \pm .57^{\mathrm{b}, \mathrm{y}}$ & $11.00 \pm 3.39^{\mathrm{c}, \mathrm{z}}$ \\
\hline 2 & $36.20 \pm 1.09^{\mathrm{a}, \mathrm{x}}$ & $21.80 \pm 4.14^{\mathrm{b}, \mathrm{y}}$ & $22.40 \pm 6.18^{\mathrm{b}, \mathrm{y}}$ & $26.57 \pm 7.95^{\text {b,y }}$ & $32.33 \pm 2.88^{\mathrm{b}, \mathrm{y}}$ & $10.80 \pm 2.94^{\mathrm{c}, \mathrm{z}}$ \\
\hline 4 & $35.80 \pm 1.64^{\mathrm{a}, \mathrm{x}}$ & $16.10 \pm 3.61^{b, y}$ & $23.50 \pm 3.80^{\mathrm{b}, \mathrm{y}}$ & $25.66 \pm 5.20^{\mathrm{b}, \mathrm{y}}$ & $26.75 \pm 6.84^{\text {b,y }}$ & $10.60 \pm 2.30^{\mathrm{c}, \mathrm{z}}$ \\
\hline 5 & $35.95 \pm 1.82^{\mathrm{a}, \mathrm{x}}$ & $21.60 \pm 2.07^{\mathrm{b}, \mathrm{y}}$ & $22.10 \pm 3.47^{\mathrm{b}, \mathrm{y}}$ & $25.33 \pm 3.14^{\mathrm{b}, \mathrm{y}}$ & $27.87 \pm 1.03^{b, y}$ & $13.60 \pm 4.03^{c, z}$ \\
\hline 6 & $36.17 \pm 1.77^{\mathrm{a}, \mathrm{x}}$ & $22.60 \pm 2.04^{b, y}$ & $23.60 \pm 2.70^{b, y}$ & $24.66 \pm 2.87^{b, y}$ & $25.75 \pm 3.94^{b, y}$ & $15.61 \pm 9.65^{\mathrm{c}, \mathrm{z}}$ \\
\hline
\end{tabular}

${ }^{a-c}$ Means with different superscripts within row differed significantly $(\mathrm{p}<0.05)$ due to treatment effect.

${ }^{x-z}$ Means with different superscripts within column differed significantly $(p<0.05)$ due to time effect. 
Table 6: Hb values of Rabbits treated with Crude Chloroform extract of Rosmarinus officinalis and those not treated (Control and Negative groups)

\begin{tabular}{|c|c|c|c|c|c|c|}
\hline \multirow{2}{*}{$\begin{array}{l}\text { Time } \\
\text { (Week) }\end{array}$} & & \multicolumn{5}{|c|}{ Groups } \\
\hline & Reference & Pre-infection & Concurrent & Post-infection & Positive control & $\begin{array}{c}\text { Negative } \\
\text { Control }\end{array}$ \\
\hline 1 & $133.00 \pm 2.54^{\mathrm{a}, \mathrm{x}}$ & $118.80 \pm 28.26^{\mathrm{a}, \mathrm{x}}$ & $98.08 \pm 5.52^{b, y}$ & $100.74 \pm 17.54^{\mathrm{a}, \mathrm{x}}$ & $102.83 \pm 11.81^{\mathrm{a}, \mathrm{x}}$ & $86.20 \pm 17.54^{\mathrm{c}, \mathrm{z}}$ \\
\hline 2 & $130.20 \pm 5.97^{\mathrm{a}, \mathrm{x}}$ & $106.78 \pm 13.76^{\mathrm{a}, \mathrm{x}}$ & $93.26 \pm 12.10^{\mathrm{b}, \mathrm{y}}$ & $105.15 \pm 19.05^{\mathrm{a}, \mathrm{x}}$ & $109.70 \pm 13.99^{\mathrm{a}, \mathrm{x}}$ & $86.00 \pm 13.96^{\mathrm{c}, \mathrm{z}}$ \\
\hline 3 & $133.20 \pm 1.78^{\mathrm{a}, \mathrm{x}}$ & $109.98 \pm 16.99^{\mathrm{a}, \mathrm{x}}$ & $94.92 \pm 13.04^{\text {by }}$ & $104.85 \pm 17.84^{\mathrm{a}, \mathrm{x}}$ & $106.66 \pm 6.35^{\mathrm{a}, \mathrm{x}}$ & $80.08 \pm 15.08^{c, z}$ \\
\hline 4 & $131.80 \pm 2.16^{\mathrm{a}, \mathrm{x}}$ & $100.60 \pm 4.50^{\mathrm{a}, \mathrm{x}}$ & $98.42 \pm 12.55^{\text {by }}$ & $109.33 \pm 19.44^{\mathrm{a}, \mathrm{x}}$ & $109.50 \pm 1.73^{\mathrm{a}, \mathrm{x}}$ & $74.94 \pm 7.07^{\mathrm{c}, \mathrm{z}}$ \\
\hline 5 & $133.60 \pm 2.60^{\mathrm{a}, \mathrm{x}}$ & $101.98 \pm 14.05^{\mathrm{a}, \mathrm{x}}$ & $93.26 \pm 12.10^{b, y}$ & $116.00 \pm 11.50^{\mathrm{a}, \mathrm{x}}$ & $111.30 \pm 3.23^{\mathrm{a}, \mathrm{x}}$ & $91.00 \pm 21.11^{\mathrm{b}, \mathrm{y}}$ \\
\hline 6 & $131.40 \pm 1.51^{\mathrm{a}, \mathrm{x}}$ & $99.48 \pm 6.86^{\mathrm{b}, \mathrm{y}}$ & $94.92 \pm 13.04^{\mathrm{b}, \mathrm{y}}$ & $104.16 \pm 13.33^{\mathrm{a}, \mathrm{x}}$ & $107.05 \pm 4.56^{\mathrm{a}, \mathrm{x}}$ & $82.78 \pm 9.43^{\mathrm{c}, \mathrm{z}}$ \\
\hline 7 & $132.00 \pm 2.34^{\mathrm{a}, \mathrm{x}}$ & $103.56 \pm 16.15^{\mathrm{a}, \mathrm{x}}$ & $98.42 \pm 12.55^{\text {b,y }}$ & $112.10 \pm 20.87^{\mathrm{a}, \mathrm{x}}$ & $106.50 \pm .57^{\mathrm{a}, \mathrm{x}}$ & $78.54 \pm 13.01^{\mathrm{c}, \mathrm{z}}$ \\
\hline
\end{tabular}

${ }^{\mathrm{a}-\mathrm{c}}$ Means with different superscripts within row differed significantly $(\mathrm{p}<0.05)$ due to treatment effect.

${ }^{\mathrm{x}-\mathrm{z}}$ Means with different superscripts within column differed significantly $(\mathrm{p}<0.05)$ due to time effect.

Table 7: RBC values of Rabbits treated with Crude Chloroform extract of Rosmarinus officinalis and those not treated (Control and Negative groups)

\begin{tabular}{|c|c|c|c|c|c|c|}
\hline \multirow{2}{*}{$\begin{array}{c}\text { Time } \\
\text { (Weeks) }\end{array}$} & & \multicolumn{5}{|c|}{ Groups } \\
\hline & Reference & Pre-infection & Concurrent & Post-infection & Positive control & Negative Control \\
\hline 1 & $4.43 \pm .24^{\mathrm{a}, \mathrm{x}}$ & $4.43 \pm .24^{\mathrm{a}, \mathrm{x}}$ & $3.84 \pm .42^{\mathrm{a}, \mathrm{x}}$ & $4.17 \pm .56^{\mathrm{a}, \mathrm{x}}$ & $4.35 \pm .30^{\mathrm{a}, \mathrm{x}}$ & $2.59 \pm .85^{\mathrm{c}, \mathrm{z}}$ \\
\hline 2 & $4.15 \pm .49^{\mathrm{a}, \mathrm{x}}$ & $3.88 \pm .29^{\mathrm{a}, \mathrm{x}}$ & $4.52 \pm .26^{\mathrm{a}, \mathrm{x}}$ & $4.26 \pm .48^{\mathrm{a}, \mathrm{x}}$ & $4.66 \pm .49^{\mathrm{a}, \mathrm{x}}$ & $2.75 \pm .70^{\mathrm{c}, \mathrm{z}}$ \\
\hline 3 & $3.99 \pm .60^{\mathrm{a}, \mathrm{x}}$ & $4.58 \pm .51^{\mathrm{a}, \mathrm{x}}$ & $3.84 \pm .42^{\mathrm{a}, \mathrm{x}}$ & $4.07 \pm .32^{\mathrm{a}, \mathrm{x}}$ & $4.35 \pm .30^{\mathrm{a}, \mathrm{x}}$ & $2.76 \pm .98^{\mathrm{c}, \mathrm{z}}$ \\
\hline 4 & $4.23 \pm .48^{\mathrm{a}, \mathrm{x}}$ & $4.43 \pm .24^{\mathrm{a}, \mathrm{x}}$ & $3.82 \pm .54^{\mathrm{a}, \mathrm{x}}$ & $4.31 \pm .71^{\mathrm{a}, \mathrm{x}}$ & $4.89 \pm .26^{\mathrm{a}, \mathrm{x}}$ & $2.59 \pm .83^{\mathrm{c}, \mathrm{z}}$ \\
\hline 5 & $4.28 \pm .24^{\mathrm{a}, \mathrm{x}}$ & $4.28 \pm .24^{\mathrm{a}, \mathrm{x}}$ & $4.16 \pm .46^{\mathrm{a}, \mathrm{x}}$ & $4.02 \pm .77^{\mathrm{a}, \mathrm{x}}$ & $4.88 \pm .37^{\mathrm{a}, \mathrm{x}}$ & $2.81 \pm .77^{\text {b.y }}$ \\
\hline 6 & $4.53 \pm .66^{\mathrm{a}, \mathrm{x}}$ & $4.17 \pm .47^{\mathrm{a}, \mathrm{x}}$ & $3.82 \pm .54^{\mathrm{a}, \mathrm{x}}$ & $4.50 \pm .37^{\mathrm{a}, \mathrm{x}}$ & $4.32 \pm .25^{\mathrm{a}, \mathrm{x}}$ & $2.46 \pm .33^{\mathrm{c}, \mathrm{z}}$ \\
\hline 7 & $3.92 \pm .42^{\mathrm{a}, \mathrm{x}}$ & $4.58 \pm .51^{\mathrm{a}, \mathrm{x}}$ & $4.16 \pm .46^{\mathrm{a}, \mathrm{x}}$ & $4.19 \pm .54^{\mathrm{a}, \mathrm{x}}$ & $4.89 \pm .26^{\mathrm{a}, \mathrm{x}}$ & $3.27 \pm .35^{\mathrm{b}, \mathrm{y}}$ \\
\hline
\end{tabular}

${ }^{\mathrm{a}-\mathrm{c}}$ Means with different superscripts within row differed significantly $(\mathrm{p}<0.05)$ due to treatment effect.

${ }^{\mathrm{x}-\mathrm{z}}$ Means with different superscripts within column differed significantly $(\mathrm{p}<0.05)$ due to time effect.

Table 8: WBC values of Rabbits treated with Crude Chloroform extract of Rosmarinus officinalis and those not treated (Control and Negative groups)

\begin{tabular}{cllllll}
\hline $\begin{array}{l}\text { Time } \\
\text { Weeks) }\end{array}$ & Reference & Pre-infection & Concurrent & Post-infection & $\begin{array}{l}\text { Positive } \\
\text { control }\end{array}$ & $\begin{array}{l}\text { Negative } \\
\text { Control }\end{array}$ \\
\cline { 2 - 6 } & & & & $4.10 \pm 1.83^{\mathrm{a}, \mathrm{x}}$ & $4.44 \pm 1.45^{\mathrm{a}, \mathrm{x}}$ \\
$\mathbf{1}$ & $4.02 \pm 1.56^{\mathrm{a}, \mathrm{x}}$ & $4.13 \pm 1.71^{\mathrm{a}, \mathrm{x}}$ & $3.85 \pm .96^{\mathrm{a}, \mathrm{x}}$ & $4.26 \pm .97^{\mathrm{a}, \mathrm{x}}$ & $2.92 \pm .00^{\mathrm{c}, \mathrm{z}}$ & $6.98 \pm 1.63^{\mathrm{b}, \mathrm{y}}$ \\
$\mathbf{2}$ & $3.07 \pm .26^{\mathrm{a}, \mathrm{x}}$ & $4.23 \pm 2.50^{\mathrm{a}, \mathrm{x}}$ & $6.30 \pm 1.23^{\mathrm{b}, \mathrm{y}}$ & $6.32 \pm 2.48^{\mathrm{b}, \mathrm{b}}$ & $2.92 \pm .00^{\mathrm{c}, \mathrm{z}}$ & $5.29 \pm 1.46^{\mathrm{b}, \mathrm{y}}$ \\
$\mathbf{3}$ & $3.24 \pm .28^{\mathrm{a}, \mathrm{x}}$ & $3.57 \pm .96^{\mathrm{a}, \mathrm{x}}$ & $4.12 \pm 1.58^{\mathrm{a}, \mathrm{x}}$ & $3.67 \pm 1.09^{\mathrm{a}, \mathrm{x}}$ & $4.84 \pm 3.84^{\mathrm{a}, \mathrm{x}}$ & $6.31 \pm 1.42^{\mathrm{b}, \mathrm{y}}$ \\
$\mathbf{4}$ & $3.84 \pm 1.66^{\mathrm{a}, \mathrm{x}}$ & $3.59 \pm 1.75^{\mathrm{a}, \mathrm{x}}$ & $3.60 \pm 1.01^{\mathrm{a}, \mathrm{x}}$ & $4.62 \pm 1.71^{\mathrm{a}, \mathrm{x}}$ & $2.92 \pm .00^{\mathrm{c}, \mathrm{z}}$ & $5.23 \pm 2.15^{\mathrm{b}, \mathrm{y}}$ \\
$\mathbf{5}$ & $3.24 \pm .28^{\mathrm{a}, \mathrm{x}}$ & $4.96 \pm 2.55^{\mathrm{a}, \mathrm{x}}$ & $4.15 \pm 1.58^{\mathrm{a}, \mathrm{x}}$ & $2.92 \pm .00^{\mathrm{c}, \mathrm{z}}$ & $2.92 \pm .00^{\mathrm{c}, \mathrm{z}}$ & $6.47 \pm 2.35^{\mathrm{b}, \mathrm{y}}$ \\
$\mathbf{6}$ & $3.24 \pm .28^{\mathrm{a}, \mathrm{x}}$ & $3.52 \pm .98^{\mathrm{a}, \mathrm{x}}$ & $4.12 \pm 1.58^{\mathrm{a}, \mathrm{x}}$ & $2.99 \pm .18^{\mathrm{a}, \mathrm{x}}$ & $2.92 \pm .00^{\mathrm{c}, \mathrm{z}}$ & $4.47 \pm 2.12^{\mathrm{a}, \mathrm{x}}$ \\
\hline
\end{tabular}

${ }^{\mathrm{a}-\mathrm{c}}$ Means with different superscripts within row differed significantly $(\mathrm{p}<0.05)$ due to treatment effect.

${ }^{\mathrm{x}-\mathrm{z}}$ Means with different superscripts within column differed significantly $(\mathrm{p}<0.05)$ due to time effect.

Table 9: The effect of Crude Chloroform extract of Rosmarinus officinalis on ALT values of Rabbits infected with T. evansi

\begin{tabular}{|c|c|c|c|c|c|c|}
\hline \multirow{2}{*}{$\begin{array}{l}\text { Time } \\
\text { (Weeks) }\end{array}$} & \multicolumn{4}{|c|}{ Groups } & \multirow[b]{2}{*}{ Positive control } & \multirow[b]{2}{*}{ Negative Control } \\
\hline & Reference & Pre-infection & Concurrent & Post-infection & & \\
\hline 1 & $43.54 \pm 5.89^{\mathrm{a}, \mathrm{x}}$ & $51.54 \pm 9.78^{\mathrm{a}, \mathrm{x}}$ & $57.54 \pm 10.37^{\mathrm{a}, \mathrm{x}}$ & $53.62 \pm 10.02^{\mathrm{a}, \mathrm{x}}$ & $46.67 \pm 5.50^{\mathrm{a}, \mathrm{x}}$ & $135.04 \pm 48.68^{\mathrm{c}, \mathrm{z}}$ \\
\hline 2 & $40.92 \pm 4.47^{\mathrm{a}, \mathrm{x}}$ & $62.92 \pm 23.45^{\text {b.y }}$ & $60.92 \pm 16.43^{\text {b,y }}$ & $62.11 \pm 18.67^{\text {b,y }}$ & $69.58 \pm 15.27^{\text {b,y }}$ & $143.07 \pm 45.36^{\mathrm{c}, \mathrm{z}}$ \\
\hline 4 & $40.57 \pm 3.24^{\mathrm{a}, \mathrm{x}}$ & $52.57 \pm 26.35^{\mathrm{a}, \mathrm{x}}$ & $50.57 \pm 17.24^{\mathrm{a}, \mathrm{x}}$ & $50.98 \pm 9.76^{\mathrm{a}, \mathrm{x}}$ & $55.04 \pm 29.75^{\mathrm{a}, \mathrm{x}}$ & $157.07 \pm 34.61^{\mathrm{c}, \mathrm{z}}$ \\
\hline 5 & $45.92 \pm 7.07^{\mathrm{a}, \mathrm{x}}$ & $57.92 \pm 13.03^{\mathrm{a}, \mathrm{x}}$ & $57.92 \pm 8.36^{\mathrm{a}, \mathrm{x}}$ & $55.58 \pm 15.97^{\mathrm{a}, \mathrm{x}}$ & $65.42 \pm 15.00^{\mathrm{a}, \mathrm{x}}$ & $129.07 \pm 15.74^{\mathrm{c}, \mathrm{z}}$ \\
\hline 6 & $40.58 \pm 5.21^{\mathrm{a}, \mathrm{x}}$ & $50.58 \pm 14.04^{\mathrm{a}, \mathrm{x}}$ & $55.99 \pm 16.51^{\mathrm{a}, \mathrm{x}}$ & $49.26 \pm 12.96^{\mathrm{a}, \mathrm{x}}$ & $52.54 \pm 19.24^{\mathrm{a}, \mathrm{x}}$ & $143.07 \pm 45.36^{\mathrm{c}, \mathrm{z}}$ \\
\hline
\end{tabular}

${ }^{\mathrm{a}-\mathrm{c}}$ Means with different superscripts within row differed significantly $(\mathrm{p}<0.05)$ due to treatment effect.

${ }^{x-z}$ Means with different superscripts within column differed significantly $(p<0.05)$ due to time effect. 
Table 10: The effect of Crude Chloroform extract of Rosmarinus officinalis on AST values of Rabbits infected with T. evansi

\begin{tabular}{|c|c|c|c|c|c|c|}
\hline \multirow{2}{*}{$\begin{array}{c}\text { Time } \\
\text { (Weeks) }\end{array}$} & & \multicolumn{5}{|c|}{ Groups } \\
\hline & Reference & Pre-infection & Concurrent & Post-infection & Positive control & Negative Control \\
\hline 1 & $93.54 \pm 27.10^{\mathrm{a}, \mathrm{x}}$ & $100.94 \pm 14.46^{\mathrm{a}, \mathrm{x}}$ & $100.94 \pm 14.46^{\mathrm{a}, \mathrm{x}}$ & $89.34 \pm 20.94^{\mathrm{a}, \mathrm{x}}$ & $93.34 \pm 20.66^{\mathrm{a}, \mathrm{x}}$ & $163.04 \pm 89.85^{\mathrm{b}, \mathrm{y}}$ \\
\hline 2 & $89.54 \pm 14.20^{\mathrm{a}, \mathrm{x}}$ & $85.54 \pm 16.54^{\mathrm{a}, \mathrm{x}}$ & $88.94 \pm 14.22^{\mathrm{a}, \mathrm{x}}$ & $87.65 \pm 14.35^{\mathrm{a}, \mathrm{x}}$ & $76.67 \pm 10.96^{\mathrm{a}, \mathrm{x}}$ & $173.07 \pm 84.36^{\mathrm{b}, \mathrm{y}}$ \\
\hline 3 & $99.54 \pm 16.33^{\mathrm{a}, \mathrm{x}}$ & $83.18 \pm 12.45^{\mathrm{a}, \mathrm{x}}$ & $94.94 \pm 15.75^{\mathrm{a}, \mathrm{x}}$ & $95.05 \pm 19.33^{\mathrm{a}, \mathrm{x}}$ & $83.34 \pm 14.73^{\mathrm{a}, \mathrm{x}}$ & $188.98 \pm 75.22^{\mathrm{b}, \mathrm{y}}$ \\
\hline 4 & $86.14 \pm 33.51^{\mathrm{a}, \mathrm{x}}$ & $100.54 \pm 18.07^{\mathrm{a}, \mathrm{x}}$ & $100.94 \pm 14.46^{\mathrm{a}, \mathrm{x}}$ & $101.34 \pm 19.81^{\mathrm{a}, \mathrm{x}}$ & $91.84 \pm 17.13^{\mathrm{a}, \mathrm{x}}$ & $167.98 \pm 84.72^{\text {b,y }}$ \\
\hline 5 & $78.14 \pm 30.97^{\mathrm{a}, \mathrm{x}}$ & $100.54 \pm 18.07^{\mathrm{a}, \mathrm{x}}$ & $92.94 \pm 18.47^{\mathrm{a}, \mathrm{x}}$ & $94.67 \pm 22.70^{\mathrm{a}, \mathrm{x}}$ & $81.84 \pm 14.15^{\mathrm{a}, \mathrm{x}}$ & $164.98 \pm 86.62^{b, y}$ \\
\hline 6 & $80.14 \pm 30.66^{\mathrm{a}, \mathrm{x}}$ & $100.54 \pm 18.07^{\mathrm{a}, \mathrm{x}}$ & $93.94 \pm 14.75^{\mathrm{a}, \mathrm{x}}$ & $101.84 \pm 22.30^{\mathrm{a}, \mathrm{x}}$ & $92.59 \pm 22.06^{\mathrm{a}, \mathrm{x}}$ & $175.98 \pm 73.45^{\mathrm{b}, \mathrm{y}}$ \\
\hline 7 & $101.74 \pm 22.13^{\mathrm{a}, \mathrm{x}}$ & $107.14 \pm 18.07^{\mathrm{a}, \mathrm{x}}$ & $83.94 \pm 7.60^{\mathrm{a}, \mathrm{x}}$ & $85.84 \pm 20.57^{\mathrm{a}, \mathrm{x}}$ & $92.84 \pm 17.07^{\mathrm{a}, \mathrm{x}}$ & $168.98 \pm 84.19^{\mathrm{b}, \mathrm{y}}$ \\
\hline
\end{tabular}

${ }^{a-c}$ Means with different superscripts within row differed significantly $(\mathrm{p}<0.05)$ due to treatment effect

${ }^{\mathrm{x}-\mathrm{z}}$ Means with different superscripts within column differed significantly $(\mathrm{p}<0.05)$ due to time effect.

Table 11: The effect of Crude Chloroform extract of Rosmarinus officinalis on ALP values of Rabbits infected with T. evansi

\begin{tabular}{|c|c|c|c|c|c|c|}
\hline \multirow{2}{*}{$\begin{array}{l}\text { Time } \\
\text { (Weeks) }\end{array}$} & \multirow[b]{2}{*}{ Reference } & \multicolumn{5}{|c|}{ Groups } \\
\hline & & Pre-infection & Concurrent & Post-infection & Positive control & Negative Control \\
\hline 2 & $69.33 \pm 17.93^{\mathrm{a}, \mathrm{x}}$ & $61.11 \pm 17.52^{\mathrm{a}, \mathrm{x}}$ & $59.53 \pm 17.32^{\mathrm{a}, \mathrm{x}}$ & $63.91 \pm 17.32^{\mathrm{a}, \mathrm{x}}$ & $62.65 \pm 23.09$ & $27.72 \pm 7.76^{\text {b,y }}$ \\
\hline 4 & $54.14 \pm 5.67^{\mathrm{a}, \mathrm{x}}$ & $46.12 \pm 11.32^{\mathrm{a}, \mathrm{x}}$ & $42.14 \pm 12.89^{\mathrm{a}, \mathrm{x}}$ & $66.17 \pm 17.81^{\mathrm{a}, \mathrm{x}}$ & $42.59 \pm 9.32^{\mathrm{a}, \mathrm{x}}$ & $27.71 \pm 7.76^{\text {b,y }}$ \\
\hline 5 & $58.11 \pm 11.14^{\mathrm{a}, \mathrm{x}}$ & $50.13 \pm 7.08^{\mathrm{a}, \mathrm{x}}$ & $50.14 \pm 12.25^{\mathrm{a}, \mathrm{x}}$ & $61.34 \pm 16.11^{\mathrm{a}, \mathrm{x}}$ & $55.09 \pm 10.17^{\mathrm{a}, \mathrm{x}}$ & $32.53 \pm 6.14^{b, y}$ \\
\hline 6 & $56.12 \pm 5.31^{\mathrm{a}, \mathrm{x}}$ & $52.14 \pm 4.02^{\mathrm{a}, \mathrm{x}}$ & $54.15 \pm 5.21^{\mathrm{a}, \mathrm{x}}$ & $52.67 \pm 11.82^{\mathrm{a}, \mathrm{x}}$ & $55.06 \pm 5.50^{\mathrm{a}, \mathrm{x}}$ & $27.73 \pm 7.76^{\mathrm{b}, \mathrm{y}}$ \\
\hline
\end{tabular}

a,b Means with different superscripts within row differed significantly $(\mathrm{p}<0.05)$ due to treatment effect.

${ }^{y, z}$ Means with different superscripts within column differed significantly $(p<0.05)$ due to time effect.

Table 12: The effect of Crude Chloroform extract of Rosmarinus officinalis on Glob values of Rabbits infected with T. evansi

\begin{tabular}{ccccccc}
\hline Time & \multicolumn{5}{c}{ Groups } \\
\cline { 2 - 6 } Weeks) & Reference & Pre-infection & Concurrent & Post-infection & Positive control & Negative Control \\
\cline { 2 - 6 } $\mathbf{1}$ & $3.01 \pm .39^{\mathrm{a}, \mathrm{x}}$ & $2.56 \pm .98^{\mathrm{a}, \mathrm{x}}$ & $3.14 \pm .60^{\mathrm{a}, \mathrm{x}}$ & $2.65 \pm .78^{\mathrm{a}, \mathrm{x}}$ & $2.34 \pm 1.15^{\mathrm{a}, \mathrm{x}}$ & $2.94 \pm .59^{\mathrm{a}, \mathrm{x}}$ \\
$\mathbf{n y y y y y y}$ & $3.14 \pm .60^{\mathrm{a}, \mathrm{x}}$ & $2.56 \pm 1.07^{\mathrm{a}, \mathrm{x}}$ & $3.05 \pm 1.41^{\mathrm{a}, \mathrm{x}}$ & $3.17 \pm .56^{\mathrm{a}, \mathrm{x}}$ & $3.00 \pm .68^{\mathrm{a}, \mathrm{x}}$ & $3.00 \pm .51^{\mathrm{a}, \mathrm{x}}$ \\
$\mathbf{3}$ & $3.38 \pm .50^{\mathrm{a}, \mathrm{x}}$ & $2.43 \pm 1.02^{\mathrm{a}, \mathrm{x}}$ & $2.90 \pm .56^{\mathrm{a}, \mathrm{x}}$ & $2.95 \pm .90^{\mathrm{a}, \mathrm{x}}$ & $2.34 \pm 1.15^{\mathrm{a}, \mathrm{x}}$ & $2.44 \pm .95^{\mathrm{a}, \mathrm{x}}$ \\
$\mathbf{4}$ & $3.43 \pm .46^{\mathrm{a}, \mathrm{x}}$ & $3.15 \pm .55^{\mathrm{a}, \mathrm{x}}$ & $3.44 \pm .40^{\mathrm{a}, \mathrm{x}}$ & $3.23 \pm .59^{\mathrm{a}, \mathrm{x}}$ & $3.28 \pm .70^{\mathrm{a}, \mathrm{x}}$ & $3.36 \pm .56^{\mathrm{a}, \mathrm{x}}$ \\
$\mathbf{5}$ & $3.49 \pm .39^{\mathrm{a}, \mathrm{x}}$ & $2.95 \pm 1.02^{\mathrm{a}, \mathrm{x}}$ & $2.93 \pm 1.38^{\mathrm{a}, \mathrm{x}}$ & $2.96 \pm .51^{\mathrm{a}, \mathrm{x}}$ & $2.31 \pm .94^{\mathrm{a}, \mathrm{x}}$ & $3.14 \pm .90^{\mathrm{a}, \mathrm{x}}$ \\
$\mathbf{6}$ & $3.25 \pm .45^{\mathrm{a}, \mathrm{x}}$ & $3.40 \pm .39^{\mathrm{a}, \mathrm{x}}$ & $2.32 \pm .64^{\mathrm{a}, \mathrm{x}}$ & $2.69 \pm .93^{\mathrm{a}, \mathrm{x}}$ & $2.61 \pm .89^{\mathrm{a}, \mathrm{x}}$ & $2.61 \pm .99^{\mathrm{a}, \mathrm{x}}$ \\
$\mathbf{7}$ & $3.34 \pm .40^{\mathrm{a}, \mathrm{x}}$ & $2.36 \pm .61$ & $2.10 \pm .31^{\mathrm{a}, \mathrm{x}}$ & $3.23 \pm .54$ & $2.62 \pm .49$ & $2.62 \pm .82^{\mathrm{a}, \mathrm{x}}$ \\
\hline
\end{tabular}

${ }^{\mathrm{a}-\mathrm{c}}$ Means with different superscripts within row differed significantly $(\mathrm{p}<0.05)$ due to treatment effect.

${ }^{\mathrm{x}-\mathrm{z}}$ Means with different superscripts within column differed significantly $(\mathrm{p}<0.05)$ due to time effect.

\section{Changes in Biochemical Values}

The mean levels of the tested blood biochemistry indices were summarized in Fig.4. In group 5 (negative group), increased levels were observed in some of the indices of ALT and AST throughout all experimental period. However, this group (negative control) showed reduced levels in serum alkaline phosphatise (ALP) during the experimental period. Furthermore, animal in negative group, serum albumin concentration decreased gradually concomitant to an increase in globulin levels leading to a decrease in the albumin-globulin ratio. There was no significant differences observed in the values of total protein, bilirubin, and plasma glucose mean values between animals treated with CCE of R.officinalis and other experimental units

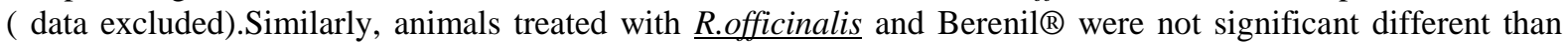
reference group in almost all tested biochemical parameters. 


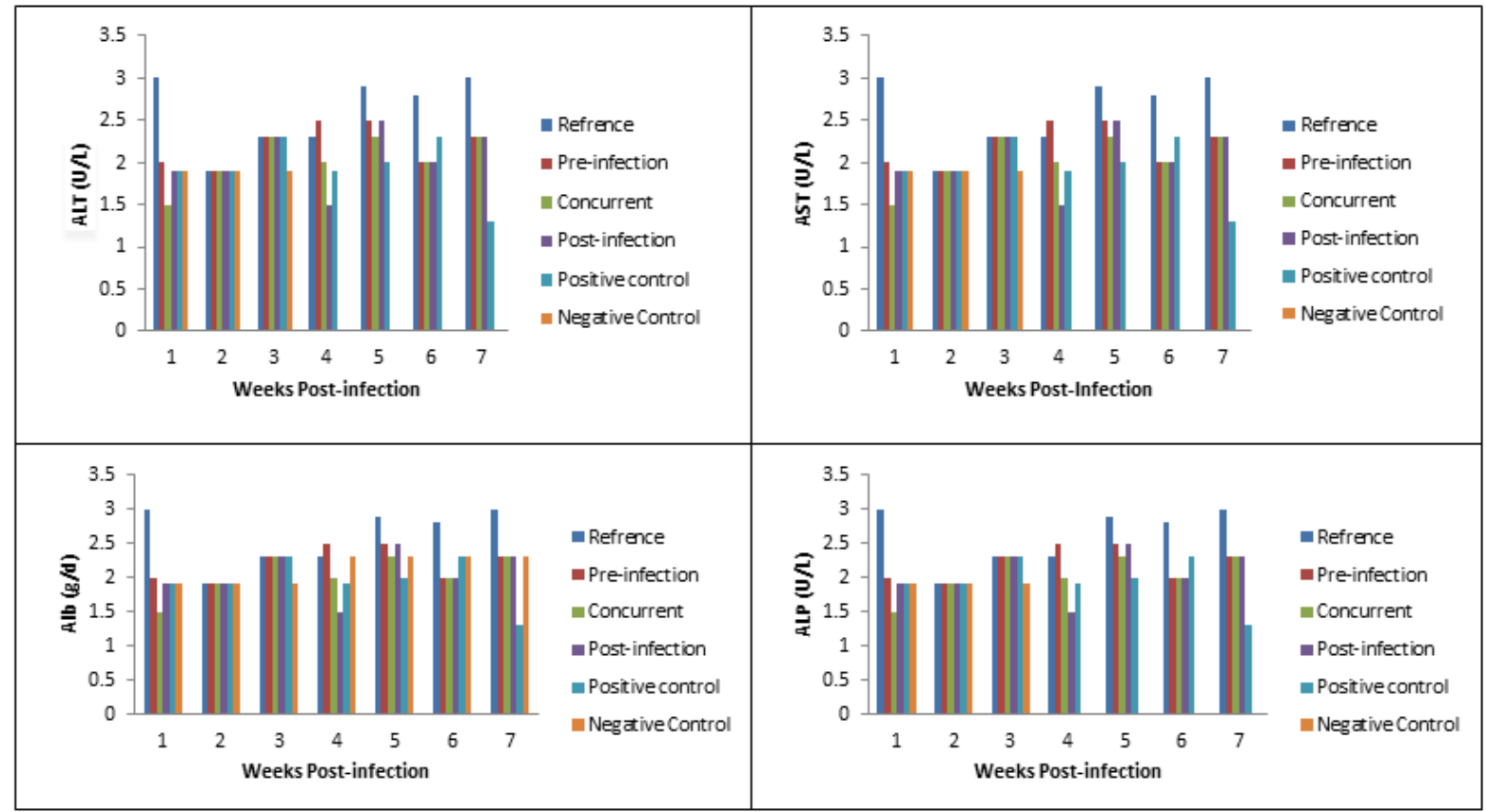

Fig. 4. Biochemical values of groups (1-6) of experimental infection of rabbits.

\section{Discussion}

This study seems to be the first investigation on the Rosmarinus officinalis as anti-trypanasomal affect in animals infected by $T$. evansi.

Since ancient times, aromatic herbs and spices have been added to different types of foods to improve the flavor and organoleptic properties. However, herbs are currently also being used in phyto-therapy and have great potential due to the different activities of secondary biomolecules, especially essential oils, which have antimicrobial, spasmolytic, carminative, hepatoprotective, antiviral, and anticarcinogenic activities (Bozin et al., 2007).

Recently, studies concerning natural products with trypanosomal activity have been relatively gaining substantial increase in wide range of disciplines. In the context of R.officinalis, most of the studies evaluated the antioxidative activity (wang, et al., 2007), anti-inflamatory properties (Benica, et al., 2010), antimicrobial activity (Tanja, et al., 2009) and it is anti-protozoal activity (Plasmodium falciparum)(Milhau et al., 1997). However, the current study examined, for the first time, the in vivo trypanosmal activities of the aerial parts of R.officinalis cultivated in Libya. Our results showed that manipulation of $T$. evansi caused the production of parasitaemia in certain stages in all groups of 1-5 that involved in the study.

In the early stages of the study, the levels of the parasitaemia observed were significantly $(p<0.05)$ associated with the tested clinical parameters; gradual loss of condition, facial oedema and ocular discharges in almost all involved groups (1-5). These clinical signs are similar to those reported earlier in other studies of experimental nature (Losos and Ikede, 1972).High levels of parasitaemia along with the rapid development of anaemia were the characteristic of this early stage. This was in agreement to Aquino et al.(1999) in dogs. In the later stage of the infection, the rabbits developed a persistent and severe anaemia while only a few parasites could be detected in the blood. This is explained by the chronic nature of the infection in these animals. Our findings showed an initial and transient parasitaemia when the condition is treated with the chloroform extract of R.officinalis, suggesting the anti-parasitic activities of R.officinalis that is comparable to the exhibited by Berenil®, a standard trypanocidal drug for treatment of trypanosomosis in animals. This was in accordance with the findings of other researchers (Atawodet al., 2000; 2005).

After development of parasitaemia, the haematological findings in the test animals showed that there is a decrease in haematological values and were indicative of anaemia. These observations were in agreement with earlier finding reported by Anene, 1987 and Mackenzie et al. 1978,that showed a progressively similar decrease in PCV and RBC in various animal species infected with trypanosomes. In this study, following treatment with crude chloroform extract of $R$. officinalis and Berenil ${ }^{\circledR}$ at 11 to 13 days after inoculation with $T$. evansi, the tested haematological parameters improved following. This improved state of anaemia is an indication of recovery from a state of cell toxicity arising from $T$. evansi infection.

Biochemically, in the negative control group there was a marked decrease in serum levels of alkaline phosphatase and albumin. This could be due to possible hepatic damage caused by the haemato-parasite induced in these animals. Unlike, the level of globulin was considerably high. This could be described to possible 
antibody production. In the negative group, hyperglobulinemia accompanied by hypoalbuminemiawas observed and is similar with the observations made in a variety of mammal hosts parasited by $T$. evansi (Singh et al., 1988; Monzon and Villavicencio, 1990; Soodanet al., 1996). In trypanosome infections, decreased serum albumin level has been reported (Katunguka-Rwakishavaet al. 1992). The decreased levels could be attributed due to hemodilution. The increased values for ALT and AST shown by negative group, in this study, may be related to the hepatic and cardiac damages.

Moreover, the reduced levels observed in the blood chemistry determinations in some of the indices such as total protein and the transaminases AST and ALT in the treated groups compared to the untreated ones reflected the beneficial effects of the plant extract.

Within the treated groups, our study showed that the extract of $R$. officinalis has no significant differences when tested pre, concurrent or post infection. Therefore, there was no significant difference among animals treated with chloroform crude extract of $R$. officinalis in terms of the biochemical and haematological values. There was no significant differences in certain parameters such as total protein, bilirubin, and plasma glucose mean values between infected and treated rabbits. Blood parasites, such as trypanosomes, depend on the host glucose for aerobic glycolysis. Apart from preventing glucose utilization by the parasites, diminazeneaceturate has been known to selectively block kinetoplast DNA replication.

\section{Conclusion}

This study demonstrated the curative properties of Rosmarinus officinalis by producing aparasitemic effect, reducing associated clinical signs of T. evansi and preventing severe high levels of AST and ALT and low values of serum alkaline phosphatase and albumin.

\section{Acknowledgments}

The researchers would like to acknowledge the efforts of the entire staff of the animal house (Rumah Haiwan) of UKM, Bangi, for their support, willingness and cooperation during the course of this research.

\section{Conflict interest statement}

There is no conflict of interest to declare.

\section{References}

[1]. Adrian, M. S., Sani, R. A., Hassan, L. \& Wong., M. T. 2010. Outbreaks of trypanosomiasis and the sero-prevalence of T. evansiin a deer breeding centre in Perak, Malaysia. Tropical Animal Health and Production, 42: 145-150.

[2]. Atawodi,SE., Bulus, T., Ibrahim, S., Ameh, DA., Noke, AJ., Mamman, M., and Galadima, M., 2003. In vitro trypanocidal effect of methanolic extract of some Nigerian Savanna plants, African Journal of Biotechnology, 2, 3117.

[3]. Bozin, B., Mimica-Dukic, N., Samojlik, I., Jovin, E., 2007. Antimicrobial and antioxidant properties of rosemary and sage (Rosmarinus officinalis L. and Salvia officinalis L., Lamiaceae) essential oils. Journal of Agricultural and Food Chemistry 55, 7879-7885.

[4]. Bauer K, Garbe D, Surburg H. In Common Fragrance and Flavor

[5]. Materials: Preparation, Properties and Uses, 2nd edn. Verlag

[6]. Chemie International: New York, 1990.

[7]. JuceliaPizzettiBeninca a, Juliana BastosDalmarco b, Moacir Geraldo Pizzolatti b, Tania Silvia Frode a, *(2011). Analysis of the antiinflammatory properties of Rosmarinus officinalis L. in mice, Food Chemistry 124, 468-475.

[8]. Katunguka-Rwakishava, E., Murray, M., Holmes, P.H.1992. The pathophisiology of ovine trypanosomiasis: haemathologicaland blood biochemical changes. Veterinary Parasitology, 45: 17-32.

[9]. Kokate C.K., Purohit A.P., Gokhale S.B., 2010. Pharmacognosy.

[10]. NiraliPrakashanPune.Blumenthal M., 1998. The complete German Commission E Monographs: Therapeutic guide to herbal medicines. Am. Bot. Coun. Austin.

[11]. Losos, G.J. and Ikede, B.O. 1972. Review of Pathology of Diseases in Domestic and Laboratory Animals Caused by Trypanosoma congolense, T.vivax, T.brucei, T.rhodesiense and T.gambiense. Veterinary Pathology,9.

[12]. Luckins A.G., Dwinger R.H. 2004. Non-tsetse-transmitted animal Trypanosomiasis. In: Maudlin I., Holmes P.H. \& Miles M.A. (Eds). The Trypanosomiases. Trowbidge: Cabi Publishing, pp.269-281.

[13]. Milhau G., Valentin A., Benoit F., Mallie M., Bastide J.M., Pelissier Y., Bessière J.M. (1997). In vitro antimalarial activity of eight essential oils. J Essent Oil Res 9: 329-333.

[14]. OIE. 2008. Manual of Diagnostic Tests and Vaccines for Terrestrial Animals www.oie.int

[15]. Oluwatuyi, M., Kaatz, G. W., \& Gibbons, S. (2004). Antibacterial and

[16]. resistance modifying activity of Rosmarinus officinalis. Phytochemistry,

[17]. 65, 3249-3254.

[18]. Tanja ROŽMAN1, Barbara JERŠEK2 (2009). Antimicrobial activity of rosemary extracts (Rosmarinus officinalis L.) against different species of Listeria, ActaagriculturaeSlovenica, 93 - 1, maj 2009 str. 51 - 58.

[19]. Peng, Y., Yuan, J., Liu, F., \& Ye, J. (2005). Determination of active components in rosemary by capillary electrophoresis with electrochemical detection. Journal of Pharmaceutical and Biomedical Analysis,39, 431-437.

[20]. W. Wang, N. Wu, Y.G. Zu *, Y.J. Fu (2008). Antioxidative activity of Rosmarinus officinalis L. essential oil compared to its main components, Food Chemistry 108, 1019-1022. 